\title{
Differential ketogenic diet-induced shift in CSF lipid/carbohydrate metabolome of pediatric epilepsy patients with optimal vs. no anticonvulsant response: a pilot study
}

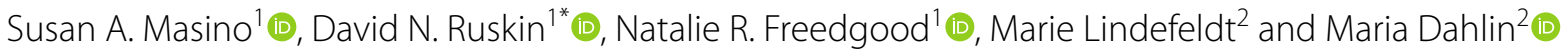

\begin{abstract}
Background: The low carbohydrate, high fat ketogenic diet can be an effective anticonvulsant treatment in some pediatric patients with pharmacoresistant epilepsy. Its mechanism(s) of action, however, remain uncertain. Direct sampling of cerebrospinal fluid before and during metabolic therapy may reveal key changes associated with differential clinical outcomes. We characterized the relationship between seizure responsiveness and changes in lipid and carbohydrate metabolites.
\end{abstract}

Methods: We performed metabolomic analysis of cerebrospinal fluid samples taken before and during ketogenic diet treatment in patients with optimal response (100\% seizure remission) and patients with no response (no seizure improvement) to search for differential diet effects in hallmark metabolic compounds in these two groups. Optimal responders and non-responders were similar in age range and included males and females. Seizure types and the etiologies or syndromes of epilepsy varied but did not appear to differ systematically between responders and non-responders.

Results: Analysis showed a strong effect of ketogenic diet treatment on the cerebrospinal fluid metabolome. Longitudinal and between-subjects analyses revealed that many lipids and carbohydrates were changed significantly by ketogenic diet, with changes typically being of larger magnitude in responders. Notably, responders had more robust changes in glucose and the ketone bodies $\beta$-hydroxybutyrate and acetoacetate than non-responders; conversely, non-responders had significant increases in fructose and sorbose, which did not occur in responders.

Conclusions: The data suggest that a differential and stronger metabolic response to the ketogenic diet may predict a better anticonvulsant response, and such variability is likely due to inherent biological factors of individual patients. Strategies to boost the metabolic response may be beneficial.

Keywords: Acetoacetate, Anticonvulsant, $\beta$-hydroxybutyrate, Cerebrospinal fluid, Glucose, Ketogenic diet, Pediatric epilepsy

*Correspondence: david.ruskin@trincoll.edu

1 Department of Psychology and Neuroscience Program, Trinity College, Hartford, CT 06106, USA

Full list of author information is available at the end of the article

\section{Introduction}

Epilepsy affects 1 in 26 people across the lifespan [1], and $25-30 \%$ of patients suffer uncontrolled seizures in spite of pharmacotherapy [2]. In addition, the incidence of side effects of anticonvulsants is high, and can lead to cessation of medications [3, 4]. Ketogenic original author(s) and the source, provide a link to the Creative Commons licence, and indicate if changes were made. The images or other third party material in this article are included in the article's Creative Commons licence, unless indicated otherwise in a credit line to the material. If material is not included in the article's Creative Commons licence and your intended use is not permitted by statutory regulation or exceeds the permitted use, you will need to obtain permission directly from the copyright holder. To view a copy of this licence, visit http://creativecommons.org/licenses/by/4.0/. The Creative Commons Public Domain Dedication waiver (http://creativeco mmons.org/publicdomain/zero/1.0/) applies to the data made available in this article, unless otherwise stated in a credit line to the data. 
diet (KD) is a metabolic therapy that employs a highfat, low-carbohydrate, moderate-protein formulation developed initially to mimic the metabolic state of fasting [5, 6]: it is established as anticonvulsant [7], antiepileptic [8], neuroprotective [9], and particularly useful in some specific diagnoses [10] and in refractory epilepsy $[11,12]$.

Fasting and adhering to a KD each produce hallmark changes in blood ketone bodies (increased) and glucose (decreased) but it has remained unclear how these changes relate to beneficial effects. For instance, blood ketone body levels do not always correlate with anticonvulsant effects [13], and in general anticonvulsant benefits of ketones versus glucose in clinical and laboratory work is mixed and still under active investigation and debate [14]. Furthermore, mechanistic links between these metabolic blood chemistry changes and anticonvulsant effects are unclear. Clearly many systemic changes occur with dietary therapy, making it difficult to sort out key mechanisms of efficacy. To date a number of studies have examined changes in blood metabolites aside from ketone bodies and glucose, typically examining a limited number of predetermined compounds to address specific hypotheses [15-21]. While cerebrospinal fluid (CSF) is significantly more difficult to collect than blood, analysis of CSF metabolites may be more relevant to KD mechanisms in epilepsy [22-25].

Metabolomics is a liquid chromatography/gas chromatography/mass spectroscopy-based technique for quantifying all detectable small molecule metabolites, such as carbohydrates, small lipids, amino acids, etc. and has been used to describe biological phenotypes in epilepsy [26] and other neurological disorders [27] as well as dietary treatments [28]. Extant metabolomics studies of serum and ketogenic diet have described compensatory adaptations in amino acid metabolism [29] and correlations between metabolites and fecal microbiota [30] in experimental rodents. In general, metabolomic analysis of CSF could be a powerful tool for discovering KD-related mechanisms. Here we present novel data based on a set of CSF samples collected twice from pediatric patients with refractory seizures - once before KD treatment and once during KD treatment. This experimental design enables each patient to serve as their own control, and thus the ability to compare baselines and changes in the metabolome to the observed clinical response. A metabolomic analysis of carbohydrates and lipids could relate the biochemical effects of the KD to the magnitude of the clinical (anticonvulsant) response, and enables a review of predictors of KD efficacy.

\section{Methods}

Ethics

The study was approved by the Ethics Committee of the Karolinska Hospital and the informed consent of the parents and, when possible, patients, was obtained.

\section{Patients}

Patients have been described in detail previously [22, 23]. Briefly, the study was part of a larger prospective open trial on the efficacy and safety of the KD in children with epilepsy. The patients were enrolled consecutively as they attended the Epilepsy Outpatient Clinic and the decision to start the KD was made.

Inclusion criteria were an age of 1-18years, pharmacologically refractory epilepsy with a prior trial of at least three antiepileptic drugs, consideration of epilepsy surgery, no medical contraindications, family and patient able to cooperate in a KD trial, and approval for performing two lumbar punctures before and during KD. Parents and other caregivers made daily notes on the number and type(s) of seizures on seizure calendars routinely used in the clinic. Parental reports were compared with the observations of seizure type and frequency made by the epilepsy nurses during hospitalization. A subgroup of 27 children were selected based on the most differentiated clinical outcome - namely those observed as seizurefree seizure and those with no observed improvement. Five of the patients became seizure-free and were classified as having "optimal response" and five patients had no detectable seizure reduction and were classified as having "no response" (see Table 1). In these ten included children metabolomics analysis with a focus on changes in carbohydrates and lipids. These patients all had variable levels of intellectual disability except for one who had autism spectrum disorder; patients were a mix of verbal and non-verbal.

\section{Study design}

This pre-KD/during KD retrospective design, performed between 1998 and 2002 at the Neuropediatric Department of the Astrid Lindgren Children's Hospital, (Karolinska Hospital), has been described in detail previously $[22,23]$. To initiate the diet, patients were hospitalized during a 4-5day stay. The 1 st CSF sampling was collected via lumbar puncture obtained immediately before diet start and the 2nd CSF sampling was performed during a 2 day inpatient stay about 3 months after KD initiation when major fine-tuning adjustments of the diet were made. During the 2nd sampling the patients were on $3: 1$ or $4: 1 \mathrm{KD}$ ratio (Table 1). All lumbar punctures were performed in the morning, around $8 \mathrm{AM}$, soon after 
Table 1 Patient demographics, sorted by clinical response

\begin{tabular}{|c|c|c|c|c|c|c|}
\hline \multicolumn{7}{|c|}{ Characteristics of 5 patients with optimal seizure reduction (no detected seizures) } \\
\hline sex & age & Epilepsy type(s) & Seizure type(s) & Etiology/syndrome & AEDs & Diet ratio \\
\hline M & 4.5 & generalized & Myoclonic, generalized T/C & Doose syndrome & VPA, CZP & $4: 1$ \\
\hline $\mathrm{F}$ & 4.8 & generalized & Myoclonic, tonic, absence & L-G syndrome & CLB & $3: 1$ \\
\hline M & 5.1 & generalized & Myoclonic, tonic & Down's syndrome & VPA, LTG & $4: 1$ \\
\hline $\mathrm{F}$ & 5.8 & generalized & Myoclonic, generalized T/C & Cortical dysplasia (polymicrogyria) & none & $3: 1$ \\
\hline $\mathrm{F}$ & 9.0 & focal & Focal without impaired awareness & Cortical dysplasia & PRI, VPA & $4: 1$ \\
\hline Mean: & 5.8 & & & & & \\
\hline \multicolumn{7}{|c|}{ Characteristics of 5 patients with no observed change in seizures } \\
\hline M & 4.0 & generalized & Myoclonic, tonic, epileptic spasms & Inverted duplicated chromosome 15, L-G syndrome & LTG, TPM & $4: 1$ \\
\hline M & 6.0 & generalized & Epileptic spasms, generalized T/C & L-G syndrome & VPA, LTG & $3: 1$ \\
\hline M & 6.3 & generalized & Myoclonic, absence, generalized T/C & Infantile spasms, L-G syndrome & VPA, CLB & $4: 1$ \\
\hline $\mathrm{F}$ & 6.7 & generalized & Generalized T/C & Unknown & LTG, PB, TPM & $4: 1$ \\
\hline M & 10.1 & generalized & Myoclonic, atonic & Agenesi corpus callosum, other CNS malformations & VPA, CZP & $4: 1$ \\
\hline Mean: & 6.6 & & & & & \\
\hline
\end{tabular}

Seizure types classified according to the International League Against Epilepsy (ILAE) classification [31]. "Diet ratio" indicates the (fat:(protein + carbohydrate)) ratio of the KD at time of 2 nd lumbar puncture. Abbreviations: $A E D$ antiepileptic drug, $T / C$ tonic/clonic, $L-G$ Lennox-Gastaux syndrome, VPA valproic acid, $C Z P$ clonazepam, $C L B$ clobazam, LTG lamotrigine, $P R I$ primidone, TPM topiramate, $P B$ phenobarbital

breakfast. During both lumbar punctures, four mL CSF was collected: samples with visible blood content were not used. Aliquots were immediately cold-centrifuged, supernatant collected, frozen, and stored at $-70^{\circ} \mathrm{C}$. Prior studies have shown that metabolites stored at this temperature are expected to remain stable for decades [32, 33]. Serum $\beta$-hydroxybutyrate $(\beta-\mathrm{HB})$ levels were measured the same morning before breakfast of the 2nd lumbar puncture and analyzed consecutively.

The diet followed a standardized protocol for the classic KD, which is a slightly modified version of the protocol of the Johns Hopkins Hospital [34]. Most patients were started at a 4:1 (fats:(proteins + carbohydrates)) ratio. A minimum of $1 \mathrm{~g} / \mathrm{kg}$ body weight per day of protein was used. At diet start supplements of vitamins and minerals were given to all patients as well as carnitine $(100 \mathrm{mg} / \mathrm{kg}$ body weight per day). All calculations of the amount of calories and the nutrient composition of the menus of the diet were made for the individual child by a specially trained dietician.

The parents were educated on all aspects of the diet by a specialized dietician, neuropediatrician, and keto nurse during a $4-5$ days inpatient stay at diet start. The parents were carefully instructed on the importance of being accurate in preparing the menus of the diet, and on keeping detailed diaries. Dietary compliance was monitored by daily testing of urine ketones at home by parents with data reported to nurses, and also by blood testing for $\beta$-hydroxybutyrate during frequent visits with the diet team consisting of nurse, dietician and neuropediatrician. Members of the team had frequent (sometimes daily) telephone consultations with parents on how to manage the diet. Also, diaries were discussed with the team during clinic visits at 1 and 3 months of treatment. Medications were not changed during the study, with the exception of slight decreases in some children.

\section{Metabolomics}

Frozen CSF samples (20 total) were sent to Metabolon (Durham, North Carolina) for metabolomics analysis in 2014. Samples were prepared and extracted. Internal standards were added to every analyzed sample as an internal quality control; samples were split into fractions for ultra-high performance liquid chromatography-tandem mass spectrometry (UPLC-MS/MS) with positive ionization, UPLC-MS/MS with negative ionization, and gas chromatography-mass spectrometry (GC-MS). The UPLC-MS/MS platform utilizes Waters Acquity UPLC with Waters UPLC BEH C18 or BEH Amide $2.1 \times 100 \mathrm{~mm}, 1.7 \mu \mathrm{m}$ columns and a ThermoScientific Q-Exactive high resolution/accurate mass spectrometer interfaced with a heated electrospray ionization (HESI-II) source and Orbitrap mass analyzer operated at 35,000 mass resolution. The GC-MS platform utilizes a Thermo-Finnigan Trace DSQ fast-scanning single-quadrupole MS using electron impact ionization operated at unit mass resolving power (scan range $50-750 \mathrm{~m} / \mathrm{z}$ ), with a 5\% diphenyl/95\% dimethyl polysiloxane fused silica column $(20 \mathrm{~m} \times 0.18 \mathrm{~mm}$ ID; 0.18 um film thickness $)$ with helium as carrier gas and a temperature ramp from $60^{\circ}$ to $340^{\circ} \mathrm{C}$ in a $17.5 \mathrm{~min}$ period. Metabolites were identified by automated comparison of the ion features in the experimental samples to a reference library of chemical standard entries that include retention time, molecular 
weight $(\mathrm{m} / \mathrm{z})$, preferred adducts, and in-source fragments as well as associated MS spectra. Identification of known chemical entities was based on comparison to metabolomic library entries of $\sim 4500$ purified standards. Raw data (ion counts) for each metabolite were scaled so that the median equaled one; missing values (nondetections) were imputed with half the minimum value. Statistical analysis (performed by Metabolon) included ANOVA, and paired and unpaired t-tests as appropriate. False discovery rate (q) was calculated for multiple comparisons: here we consider $\mathrm{q}<0.10$ to indicate high confidence in a result, and $0.10<\mathrm{q}<0.20$ to indicate moderate confidence.

\section{Results}

The patient group included all five children with $100 \%$ seizure reduction ("optimal response") and all five patients with no seizure reduction; demographics of the patient group are given in Table 1. Patients with optimal seizure response and no observed seizure remission to KD treatment had similar age ranges and included both males and females. Seizure types and the etiology of the epilepsy or epilepsy syndrome varied and did not appear to differ systematically between patients with an optimal vs. no observable clinical response. Similarly, anticonvulsant drug treatment did not appear to differ systematically between the two groups, nor did KD ratio. Statistical analysis was not performed on these factors due to variability, overlap in diagnostic categories and small sample size.

In CSF samples from these patients, 271 metabolites were identified. We focused on analyzing CSF compounds in the lipid and carbohydrate families (49 and 25 compounds detected, respectively. In the lipid family 25/49 compounds changed significantly (comparing baseline to during KD) in patients with an optimal vs. no observable clinical response; in the carbohydrate family, $15 / 25$ changed significantly in optimal or no observable clinical response (Table 2). Considering both families together, there was a strong trend $(p=0.066)$ for more compounds to change baseline-to-KD in patients with an optimal vs. no observable clinical response.

CSF lipids and their metabolites were mostly increased by KD treatment (Table 2), including the hydroxylated derivatives and carnitine esters of medium- and short-chain fatty acids. The ketone bodies acetoacetate and $\beta$-hydroxybutyrate were increased many-fold, and although the ketone body acetone was not itself detected, its metabolite 1,2-propanediol [35] was detected and elevated significantly. There were large increases in trimethylamine $\mathrm{N}$-oxide (a gut microbiotic metabolite of dietary carnitine and lecithin) and glycocholate (a gut microbiota-modified bile acid). There were decreases in methylmalonylcarnitine (involved in fatty acid synthesis) and glycerol 3-phosphate (a triglyceride metabolite). Many of these changes were significant only in optimal responders. Regarding compounds that were significantly changed in both patients with an optimal vs. no observable clinical response (e.g. hexanoyl- and acetyl-carnitine, 3-hydroxyoctanoate, trimethylamine $\mathrm{N}$-oxide), examination of the fold changes in means reveals larger magnitude changes in optimal responders.

Not surprisingly, since a KD greatly reduces dietary carbohydrates, CSF sugars and their metabolites/derivatives during KD therapy changed greatly from baseline to during KD (Table 3). Some carbohydrates were decreased, including glucose (significant only in optimal responders) and the glycemic indicator 1,5-anhydroglucitol [36]. A number of hexoses and pentoses, however, were increased. Elevated pyruvate in brain was previously noted in rodents eating a KD [37]. Notably, sorbose and fructose were significantly increased only in patients with no clinical response.

As for between-subjects comparisons of CSF metabolites in patients with an optimal vs. no clinical response during KD treatment, no compounds survived application of a false-discovery rate correction. However, several showed differences that were significant with uncorrected t-tests (Table 4), and these demonstrated a pattern that supported the within-subjects comparison. Ketone bodies and some fatty acids and hydroxylated fatty acids were several-fold higher in patients with optimal vs. no clinical response. In addition, glucose, sorbose, and fructose were lower in optimal responders.

Between-subject comparisons of CSF metabolites in patients with optimal vs. no clinical response in baseline samples revealed only one compound that was significant with an uncorrected t-test at $\mathrm{p}<0.05$ : the hexose mannose, higher in patients with no clinical response (Table 4).

Statistical analysis was further focused on the hallmark changes in biochemistry within the lipid and carbohydrate families, namely the ketone bodies and glucose. We applied two-by-two ANOVAs to these CSF metabolites with clinical response to $K D$ and time as factors. There were no baseline differences between the groups (locants are overlapping in Fig. 1). As expected, ketone bodies ( $\beta$-hydroxybutyrate and acetoacetate) were elevated and glucose was lowered during KD treatment; however, all these responses were significantly smaller in the patients with no clinical response vs. those with optimal response (Fig. 1). Serum levels of $\beta$-hydroxybutyrate during KD were also 
Table 2 Ketogenic diet-induced changes in lipids

\begin{tabular}{|c|c|c|c|}
\hline \multirow[b]{2}{*}{ Compound Family } & \multirow[b]{2}{*}{ Compound } & \multicolumn{2}{|c|}{ During diet/Prediet } \\
\hline & & optimal response & no response \\
\hline \multirow[t]{3}{*}{ Ketone Bodies } & 1,2-propanediol & $29.16^{* * *}$ & $7.47^{* *}$ \\
\hline & Acetoacetate & $130.8^{* * *}$ & $67.5^{* * *}$ \\
\hline & $\beta$-hydroxybutyrate & $175.5^{* * *}$ & $57.4^{* * *}$ \\
\hline \multirow[t]{6}{*}{ Acyl Carnitines } & Propionyl-carnitine & $1.50^{*}$ & 1.48 \\
\hline & Acetyl-carnitine & $5.19^{* * *}$ & $3.89^{*}$ \\
\hline & Butyryl-carnitine & $5.51^{* *}$ & 2.40 \\
\hline & Octanoyl-carnitine & $9.43^{* * *}$ & 5.44 \\
\hline & Hexanoyl-carnitine & $16.09^{* * *}$ & $4.73^{* *}$ \\
\hline & 3-hydroxybutyryl-carnitine & $23.63^{* * *}$ & $15.84^{* *}$ \\
\hline \multirow[t]{3}{*}{ Carnitine Metabolism } & Carnitine & 0.99 & 1.19 \\
\hline & Deoxycarnitine & 2.00 & 1.35 \\
\hline & 3-dehydrocarnitine & $61.96^{* * *}$ & $108.5^{* * *}$ \\
\hline \multirow[t]{6}{*}{ Monohydroxy Fatty Acids } & 2-hydroxyhexanoate & 1.28 & 2.10 \\
\hline & 3-hydroxypropanoate & 2.35 & 1.46 \\
\hline & Y-hydroxybutyrate & $3.40^{* * *}$ & $2.06^{* *}$ \\
\hline & 5-hydroxyhexanoate & $6.48^{*}$ & $2.29^{*}$ \\
\hline & 3-hydroxyoctanoate & $10.35^{* * *}$ & $3.48^{* * *}$ \\
\hline & 3-hydroxysebacate & $22.12^{* *}$ & 6.70 \\
\hline \multirow[t]{6}{*}{ Medium Chain Fatty Acids } & Nonanoic acid & 0.97 & 1.19 \\
\hline & Dodecanoic acid & 1.05 & 0.98 \\
\hline & Decanoic acid & $1.14^{*}$ & 1.12 \\
\hline & Octanoic acid & 1.90 & 1.15 \\
\hline & Heptanoic acid & 2.24 & 0.73 \\
\hline & Hexanoic acid & 3.29 & 1.49 \\
\hline \multirow[t]{4}{*}{ Phospholipid Metabolism } & Choline & 0.96 & 0.94 \\
\hline & Phosphoethanolamine & 1.02 & 1.26 \\
\hline & Choline phosphate & 1.47 & 2.50 \\
\hline & Trimethylamine $\mathrm{N}$-oxide & $715.1^{* *}$ & $375.3^{* *}$ \\
\hline Amide Fatty Acid & Oleamide & 0.99 & 16.48 \\
\hline \multirow[t]{2}{*}{ Dicarboxylate Fatty Acids } & 2-hydroxyglutarate & 2.05 & 1.07 \\
\hline & CMPF & $8.81^{*}$ & 2.61 \\
\hline \multirow[t]{2}{*}{ Triglyceride Metabolism } & Glycerol 3-phosphate & $0.82^{* *}$ & 0.86 \\
\hline & Glycerol & 1.22 & 0.89 \\
\hline \multirow[t]{4}{*}{ Lysophospholipids } & 1-oleoyl-GPC (18:1) & $0.46^{*}$ & 2.27 \\
\hline & 1-docosahexaenoyl-GPE (22:6) & 0.93 & 0.84 \\
\hline & 1-oleoyl-GPE (18:1) & 1.29 & 1.51 \\
\hline & 1-palmitoyl-GPC (16:0) & 3.16 & 1.90 \\
\hline \multirow[t]{2}{*}{ Inositol Metabolism } & Scyllo-inositol & 0.84 & 0.81 \\
\hline & Myo-inositol & 1.12 & 0.81 \\
\hline \multirow[t]{2}{*}{ Amino Fatty Acids } & 2-aminoheptanoate & 1.38 & 1.67 \\
\hline & 2-aminooctanoate & 631.4 & $9.24^{*}$ \\
\hline Fatty Acid Synthesis & 2-methylmalonylcarnitine & $0.64^{* * *}$ & $0.69^{*}$ \\
\hline Polyunsaturated Fatty Acid & Docosahexaenoate (22:6n3) & 2.05 & 1.07 \\
\hline Primary Bile Acid Metabolism & Glycocholate & $12.08^{*}$ & 2.44 \\
\hline Sphingolipid Metabolism & Palmitoyl sphingomyelin (d18:1/16:0) & $2.66^{*}$ & 1.73 \\
\hline \multirow[t]{2}{*}{ Corticosteroids } & Cortisone & $0.73^{* *}$ & 1.19 \\
\hline & Cortisol & 0.98 & 1.65 \\
\hline Androgenic Steroid & Androstenediol $(3 \beta, 17 \beta)$ disulfate & 6.31 & 6.02 \\
\hline Sterols & $7-a-H O C A$ & 0.95 & $0.88^{*}$ \\
\hline
\end{tabular}

Ratios are fold changes from during KD compared to before KD. Compounds are grouped by compound family, and by optimal response ratio within that family. Significant increases are indicated with bold, significant decreases with italics. False-discovery rates: ${ }^{*} 0.10<\mathrm{q}<0.20,{ }^{* *} 0.05<\mathrm{q}<0.10,{ }^{* * *} \mathrm{q}<0.05$. 7-HOCA -7 -alphahydroxy-3-oxo-4-cholestenoate, CMPF - 3-carboxy-4-methyl-5-propyl-2-furanpropanoate, GPC glycerol-3-phosphocholine, GPE glycero-3-phosphoethanolamine 
Table 3 Diet-induced changes in carbohydrates

\begin{tabular}{|c|c|c|c|}
\hline \multirow[b]{2}{*}{ Compound Family } & \multirow[b]{2}{*}{ Compound } & \multicolumn{2}{|c|}{ During diet/Prediet } \\
\hline & & optimal response & no response \\
\hline \multirow[t]{5}{*}{ Glycolysis } & 1,5-anhydroglucitol & $0.57^{* *}$ & $0.47^{* *}$ \\
\hline & Glucose & $0.83^{* * *}$ & 0.93 \\
\hline & Lactate & 0.96 & 1.05 \\
\hline & Glycerate & 1.12 & 0.99 \\
\hline & Pyruvate & $1.18^{* * *}$ & 1.93 \\
\hline \multirow[t]{6}{*}{ Hexose Metabolism } & Galactitol (dulcitol) & $0.67^{* *}$ & 0.87 \\
\hline & Sorbose & $0.71^{*}$ & $8.04^{* *}$ \\
\hline & Sorbitol & 1.11 & 1.27 \\
\hline & Fructose & 1.13 & $1.46^{* *}$ \\
\hline & Mannose & $1.52^{* * *}$ & $1.35^{* * *}$ \\
\hline & Mannitol & $1.66^{* * *}$ & $1.70^{* *}$ \\
\hline \multirow[t]{9}{*}{ Pentose Metabolism } & Threitol & $0.61^{*}$ & $0.74^{*}$ \\
\hline & Xylitol & 0.94 & 0.92 \\
\hline & Ribitol & 1.04 & 0.97 \\
\hline & Xylose & 1.12 & 1.04 \\
\hline & Ribose & 1.18 & 1.12 \\
\hline & Ribulose & $1.37^{*}$ & 1.16 \\
\hline & Xylonate & $1.38^{* *}$ & 1.15 \\
\hline & Arabitol & $1.54^{* * *}$ & $1.48^{* *}$ \\
\hline & Arabinose & $2.38^{* * *}$ & $1.63^{* * *}$ \\
\hline \multirow[t]{3}{*}{ Aminosugar Metabolism } & Erythronate & $0.84^{*}$ & $0.77^{* *}$ \\
\hline & $\mathrm{N}$-acetylneuraminate & 0.98 & 0.93 \\
\hline & Glucuronate & 1.04 & 1.04 \\
\hline Advanced Glycation End-product & Erythrulose & 1.3 & $0.58^{*}$ \\
\hline
\end{tabular}

Ratios are fold changes from during KD compared to before KD. Compounds are grouped by compound family, and by optimal response ratio within that family. Significant increases are indicated with bold, significant decreases with italics. False-discovery rates: ${ }^{*} 0.10<\mathrm{q}<0.20,{ }^{* *} 0.05<\mathrm{q}<0.10,{ }^{* * *} \mathrm{q}<0.05$

significantly lower in patients with no clinical response $(3.2 \pm 0.4 \mathrm{mM}) \quad$ vs. those with optimal response $(5.2 \pm 0.1 \mathrm{mM}, p<0.01)$, expected as CSF ketone body levels track blood levels [38-42].

Follow-up data are available on all patients. In the 5 patients who rapidly acquired seizure freedom, "optimal responders", the KD was continued between 2 years and 4.6 years. In three of these the KD was tapered and several years after discontinuation of the diet they were still seizure-free. In two patients, however, seizures came back during tapering: in one there are very few seizures but in the other the seizures were as bad as before KD. In the five patients with no seizure response, the diet was tapered after 3-4 months in three patients, but parents of two patients persevered with the KD because of improved communication and better alertness. Dietary treatment was continued for 2-3 years with these behavioral improvements persisting.

\section{Discussion}

We characterized KD-induced changes in the CSF lipid/ carbohydrate metabolome in pediatric epilepsy patients with the most distinct clinical behavioral responses at 3 months: either no detectable seizures (optimal response) or no detectable change in the frequency of refractory seizures. A major finding from this analysis, supported by longitudinal and between-subjects analyses, is that those patients who had an optimal response had a stronger metabolic response to the KD than those patients who showed no seizure improvement. This effect was evident by a larger number of KD-changed compounds and a larger magnitude change in most of those compounds in optimal responders - including glucose and the ketone bodies. While this pattern might be interpreted to indicate that the patients with no clinical response were less compliant, there is some evidence to suggest this is not true. For example, diet compliance was 
Table 4 Between-subject differences, optimal v. no response, on and before ketogenic diet

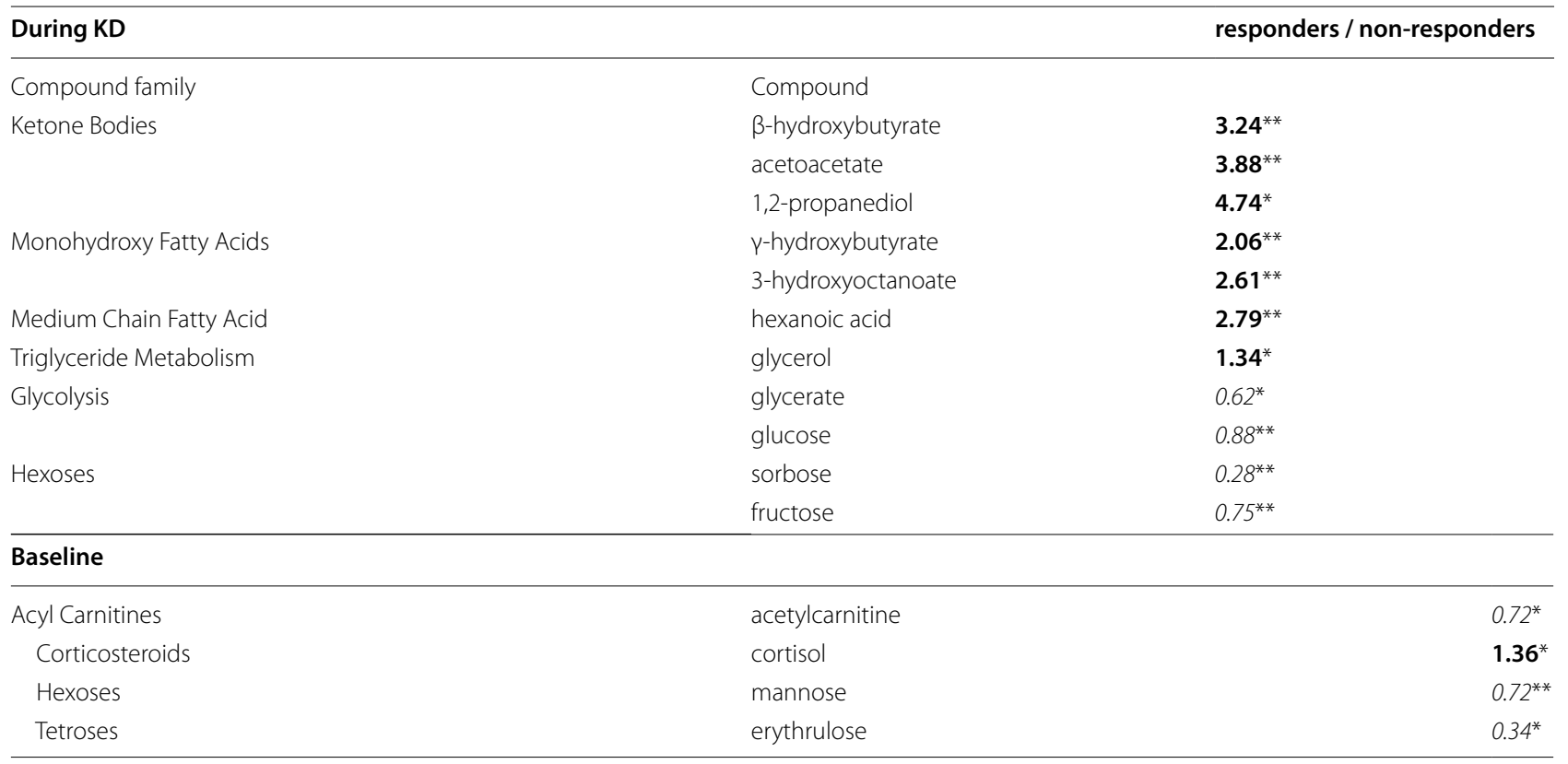

Ratios are fold difference between patients with an optimal vs. no clinical response. Only compounds with $p<0.10$ are listed. ${ }^{*} 0.05<p<0.10,{ }^{* *} p<0.05$

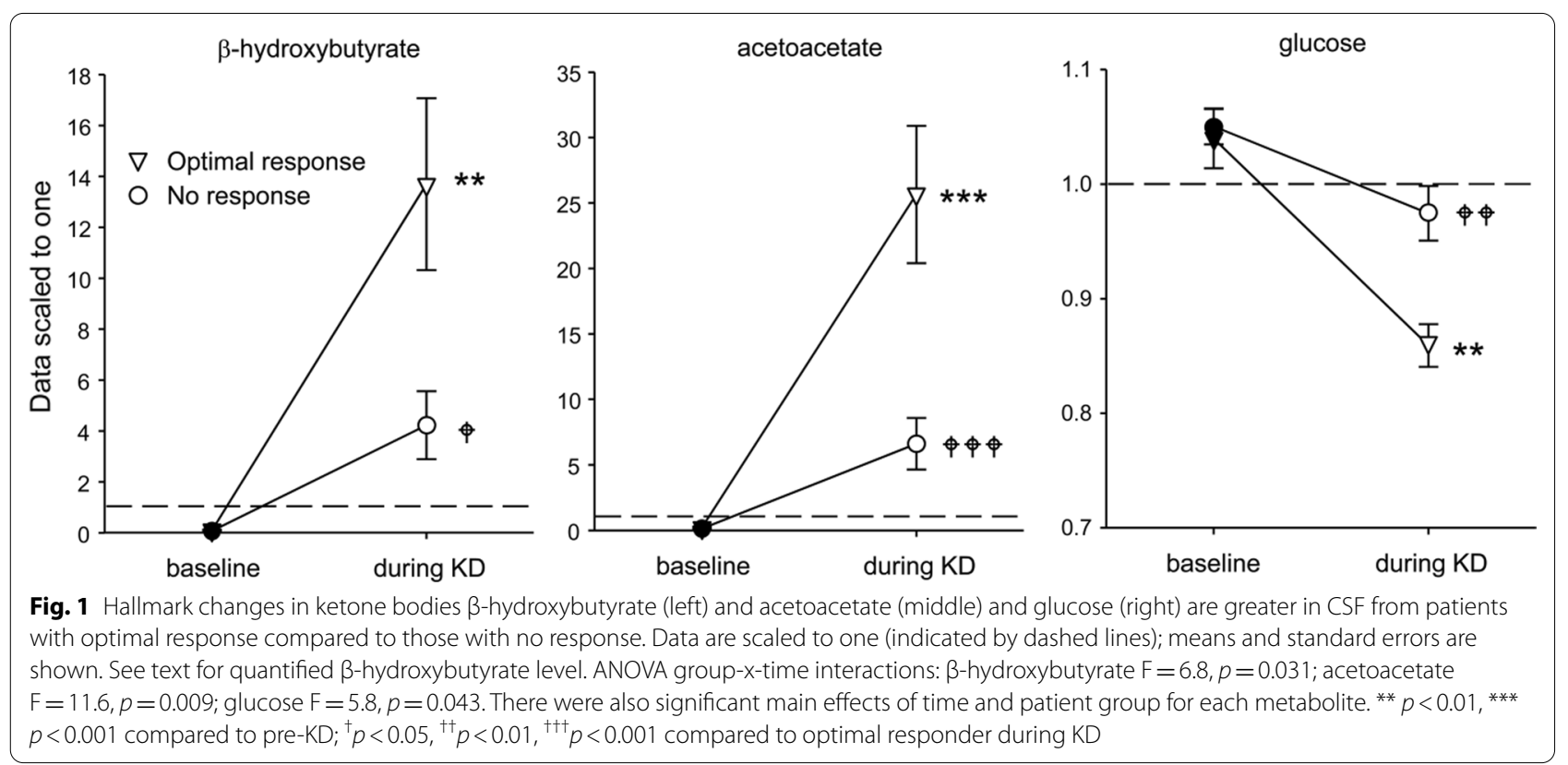

described as good by caretakers and medical personnel. Furthermore, all patients with no clinical response (as well those with optimal response) had striking elevations of CSF ketone bodies during KD compared to pre-diet (and high blood ketone bodies during KD), confirming adherence to a KD that precipitated a shift to ketonebased metabolism. Thus, these differences may be due to inherent biological variability in metabolic response. We cannot rule out, however, that some external dietary factors (exact ratio of KD administered, types of fat or of protein, etc.) could be involved.

Of paramount interest is the identification of compounds that could be mechanistically involved in the anticonvulsant effect of a robust metabolic response. The 
eponymous products of a KD are an obvious candidate [14]. Beyond being a more efficient fuel for in oxidative metabolism and mitochondrial health, ketone bodies are putative signaling molecules in several pathways that should improve the state of hyperexcitable brain tissue, including inhibitory ion channels [43], vesicular glutamate release [44], inflammation [45], nicotinamide adenine dinucleotide oxidation state [46], and histone deacetylases [47]. It was realized in the earliest KD studies that some threshold level of blood ketone bodies needed to be reached for beneficial outcomes [48, 49]. Beyond that, however, the connection of elevated blood ketone bodies and anticonvulsant effects clinically has been controversial, described as correlated [50-52] or not [53]. Studies have noted that some individual patients benefit despite not being in ketosis [54-56] or have described a correlation only at certain lengths of $\mathrm{KD}$ treatment $[55,57]$.

Besides elevating brain ketone body levels via the liver, low glucose appears to have direct effects in brain. Mildly lowered glucose promotes an autocrine inhibition in pyramidal neurons in vitro based on the neuromodulator adenosine [58]. This same mechanism is invoked by KD treatment [59] and is anti-seizure [60]. Similarly, partial block of glycolysis with 2-deoxyglucose tempers epileptiform activity in vitro [61] and seizures in vivo in some models [62]. Low glycemic index diets can be anticonvulsant in the absence of ketosis [63, 64], and low glucose rather than elevated ketone bodies seems to underlie the anticonvulsant action of caloric restriction [65]. At the other end of the glycolytic pathway, elevated pyruvate was significant in optimal responders only, which may indicate decreased usage due to increased acetyl-CoA production from ketone bodies.

Acetyl-carnitine, associated with dietary red meat [28] is involved in synthesis of acetyl-CoA. Although little work has been done with seizures directly [66], this molecule is thought to be neuroprotective, neurotrophic, and antioxidant. Notably, acetyl-carnitine is well-tolerated as a dietary supplement [67].

Conversely, this metabolomics analysis revealed some elevated compounds that may be mechanistically detrimental to the efficacy of a KD. KD-related elevations in fructose and sorbose occur in patients with no clinical response and not those with optimal response. Because fructose and sorbose can be metabolized to fructose6-phosphate, these KD-related changes could augment glycolytic metabolites downstream of glucose. Combined with a smaller reduction in glucose itself in patients with no clinical response, this pattern might indicate that substantial amounts of acetyl-coA are still entering the Kreb's cycle from the glycolytic pathway. This state may offset the enhanced efficiency of adenosine triphosphate production during ketolysis, undermining the beneficial effects of the KD-related metabolic state presumably occurring with full force in optimal responders.

Many detected compounds likely reflect a stronger metabolic response to the $\mathrm{KD}$ in optimal responders without being mechanistically involved in a better anticonvulsant response. For instance, increases in trimethylamine $\mathrm{N}$-oxide is to be expected in a diet rich in meat, fish, and eggs; changes in glycocholate and methylmalonyl carnitine comport with a high fat diet; decreased glycerol 3-phosphate could be due to increased shunting into glycolysis (via dihydroxyacetone phosphate) to augment this underpowered pathway - none of these metabolites are thought to have signaling properties in brain. 3-hydroxyoctanoate is a signaling molecule with specific receptors expressed in adipocytes but not brain [68]. $\gamma$-hydroxybutyrate also acts on specific receptors (GPR172A), and is used exogenously as an abused hypnotic/sedative drug. However, antagonism of GPR172A is anticonvulsant; conversely, activation is used to produce an animal model of absence seizures [69] making higher $\gamma$-hydroxybutyrate unlikely to be involved in a better anticonvulsant response to the KD. It was hypothesized that elevated $\beta$-hydroxybutyrate might mediate the mild euphoria found during fasting and sometimes during KD [70] by acting pharmacologically like $\gamma$-hydroxybutyrate; however, elevated $\gamma$-hydroxybutyrate itself may underlie this effect.

These findings can be related to prior clinical studies of the plasma metabolome, and some changes found in plasma were not present in these CSF samples. For instance, KD did not elevate cortisol in CSF, whereas it has been reported to do so in plasma; these latter studies were, however, in rheumatoid arthritis patients [19, 53]. Cappuccio et al. reported elevated plasma hydroxybutyryl- and acetyl-carnitine and lower methylmalonylcarnitine in KD-treated glucose transporter 1 deficient patients [16], similar to the present results. Also, Schoeler et al. demonstrated a stronger $\beta$-hydroxybutyrate response to KD treatment in patients with optimal clinical response vs. those with no response; however, they also reported that optimal responders had higher baseline CSF $\beta$-hydroxybutyrate and octanoyl- and acetylcarnitine [21]. We did not replicate those findings, and in fact showed a trend for lower initial acetyl-carnitine in optimal responders (and a significant increase during KD was also specific to optimal responders - showing a strong regulation by KD). These discrepancies could be due to true differences between plasma and CSF, or might reflect dissimilar definitions of patients with optimal response or no clinical response. 
We acknowledge some limitations to the present study. The number of subjects is low, which reduces statistical power. Also, we are unaware of any clinical study that describes effects of the presently-used anticonvulsants on CSF levels of our metabolites of interest, so if and how these drugs had any effect remains unknown. Last, metabolomics was not performed on plasma, which was collected but lost in a freezer accident. This could have indicated correlations between CSF and plasma and possibly identified blood-born predictors of clinical response.

\section{Conclusions}

In a small but valuable clinical cohort with pre- and post-KD CSF sampling, we found a more effective anticonvulsant response correlated with a stronger central metabolic response at 3 months of KD treatment. Such samples present a special opportunity as studies with sequential lumbar punctures in pediatric patients are rare, but allow for within-subjects analysis. This study extends and complements prior work on monoamine and amino acid neurotransmitters in CSF [22, 23]. Focused studies of specific metabolites are valuable, but the unpredicted changes found here (such as KD-elevated levels of hexoses only in non-reponders) highlight the importance of casting a wide net. Further work is needed and could determine more quantitatively if a strong central metabolic response early in KD treatment predicts an ultimately effective anticonvulsant outcome, information that may be useful in decisions about treatment adjuvants, adjustments and/or continuation. In addition, a blood-based correlate that predicts efficacy would be extremely valuable because CSF collection is necessarily invasive. It is worth noting that some of the relevant metabolites (ketone bodies, 1,2-propanediol, glycerol 3-phosphate) are detectable with ${ }^{1} \mathrm{H}$ or ${ }^{31} \mathrm{P}$ nuclear magnetic resonance spectroscopy. For those whose poor anticonvulsant response may due to inherent biological factors, on possibility is that KD treatment could be amplified by manipulating hallmark metabolic changes directly - for example supplementing with relevant molecules, e.g. ketone bodies (via precursors such as ketone esters, medium chain triglycerides, or coconut oil) or acetyl-carnitine.

\footnotetext{
Abbreviations

7-HOCA: 7-alpha-hydroxy-3-oxo-4-cholestenoate; AED: Antiepileptic drug; CLB: Clobazam; CMPF: 3-carboxy-4-methyl-5-propyl-2-furanpropanoate; CSF: Cerebrospinal fluid; CZP: Clonazepam; GC-MS: Gas chromatography-mass spectrometry; GPC: Glycero-3-phosphocholine; GPE: Glycero-3-phosphoethanolamine; HESI: Heated electrospray ionization; KD: Ketogenic diet; L-G: Lennox-Gastaux syndrome; LTG: Lamotrigine; PB: Phenobarbital; PRI: Primidone; T/C:Tonic/clonic; TPM: Topiramate; UPLC-MS/MS: Ultra-high performance liquid chromatography-tandem mass spectrometry; VPA: Valproic acid.
}

Acknowledgements

The authors thank Julia R. Duggan and Daisuke S. Kasamatsu for assistance with data analysis.

\section{Authors' contributions}

Conceptualization, sample collection, patient interactions: MD, ML. Analysis: DNR, NRF, SAM. Writing: MD, DNR, SAM. Editing: MD, DNR, SAM. The author(s) read and approved the final manuscript.

\section{Funding}

SAM (NIH NS066392, NS065957), DNR (NIH AT008742).

\section{Availability of data and materials}

The dataset produced and analyzed in this study is available from the corresponding author on reasonable request.

\section{Ethics approval and consent to participate}

The study was approved by the Ethics Committee of the Karolinska Hospital and the informed consent of the parents and, when possible, patients, was obtained.

\section{Consent for publication \\ Not applicable.}

\section{Competing interests}

The authors declare that they have no competing interests.

\section{Author details}

${ }^{1}$ Department of Psychology and Neuroscience Program, Trinity College, Hartford, CT 06106, USA. ${ }^{2}$ Neuropediatric Department, Astrid Lindgren Children's Hospital, Karolinska Hospital, Stockholm, Sweden.

Received: 6 August 2020 Accepted: 21 November 2020

Published online: 01 March 2021

\section{References}

1. Hauser WA. Epilepsy frequency, causes, and consequences. New York: Demos; 1990

2. Nadkarni S, LaJoie J, Devinsky O. Current treatments of epilepsy. Neurology. 2005;64:S2-11.

3. Chen B, Detyniecki K, Choi H, Hirsch L, Katz A, Legge A, et al. Psychiatric and behavioral side effects of anti-epileptic drugs in adolescents and children with epilepsy. Eur J Paediatr Neurol. 2017;21:441-9.

4. Toledano R, Gil-Nagel A. Adverse effects of antiepileptic drugs. Semin Neurol. 2008;28:317-27.

5. Wilder RM. The effects of ketonemia on the course of epilepsy. Mayo Clin Bull. 1921;2:307

6. Wilder RM. High fat diets in epilepsy. Mayo Clin Bull. 1921;2:308.

7. Bough KJ, Rho JM. Anticonvulsant mechanisms of the ketogenic diet. Epilepsia. 2007;48:43-58.

8. Lusardi TA, Akula KK, Coffman SQ, Ruskin DN, Masino SA, Boison D. Ketogenic diet prevents epileptogenesis and disease progression in adult mice and rats. Neuropharmacology. 2015;99:500-9.

9. Gasior M, Rogawski MA, Hartman AL. Neuroprotective and disease-modifying effects of the ketogenic diet. Behav Pharmacol. 2006;17:431-9.

10. Kossoff EH, Zupec-Kania BA, Auvin S, Ballaban-Gil KR, Christina Bergqvist AG, Blackford R, et al. Optimal clinical management of children receiving dietary therapies for epilepsy: Updated recommendations of the International Ketogenic Diet Study Group. Epilepsia Open. 2018;3:175-92.

11. HallböökT, Kohler S, Rosén I, Lundgren J. Effects of ketogenic diet on epileptiform activity in children with therapy resistant epilepsy. Epilepsy Res. 2007:77:134-40.

12. Kim DY, Rho JM. The ketogenic diet and epilepsy. Curr Opin Clin Nutr Metab Care. 2008;11:113-20.

13. Bough KJ, Chen RS, Eagles DA. Path analysis shows that increasing ketogenic ratio, but not b-hydroxybutyrate, elevates seizure threshold in the rat. Dev Neurosci. 1999;21:400-6. 
14. Simeone TA, Simeone KA, Stafstrom CE, Rho JM. Do ketone bodies mediate the anti-seizure effects of the ketogenic diet? Neuropharmacology. 2018:133:233-41.

15. Ahola S, Auranen M, Isohanni P, Niemisalo S, Urho N, Buzkova J, et al. Modified Atkins diet induces subacute selective ragged-red-fiber lysis in mitochondrial myopathy patients. EMBO Mol Med. 2016;8:1234-47.

16. Cappuccio G, Pinelli M, Alagia M, Donti T, Day-Salvatore DL, Veggiotti $P$, et al. Biochemical phenotyping unravels novel metabolic abnormalities and potential biomarkers associated with treatment of GLUT1 deficiency with ketogenic diet. Plos One. 2017;12:e0184022.

17. Christodoulides SS, Neal EG, Fitzsimmons G, Chaffe HM, Jeanes YM, Aitkenhead $\mathrm{H}$, Cross $\mathrm{JH}$. The effect of the classical and medium chain triglyceride ketogenic diet on vitamin and mineral levels. J Hum Nutr Diet. 2012;25:16-26.

18. Forsythe CE, Phinney SD, Feinman RD, Volk BM, Freidenreich D, Quann E, et al. Limited effect of dietary saturated fat on plasma saturated fat in the context of a low carbohydrate diet. Lipids. 2010;45:947-62.

19. Fraser DD, Whiting S, Andrew RD, Macdonald EA, Musa-Veloso K, Cunnane SC. Elevated polyunsaturated fatty acids in blood serum obtained from children on the ketogenic diet. Neurology. 2003;60:1026-9.

20. Neth BJ, Mintz A, Whitlow C, Jung Y, Sai KS, Register TC, et al. Modified ketogenic diet is associated with improved cerebrospinal fluid biomarker profile, cerebral perfusion, and cerebral ketone body uptake in older adults at risk for Alzheimer's disease: a pilot study. Neurobiol Aging. 2020;86:54-63.

21. Schoeler, NEB, G., A Yuen, AD Kapelner, SJR Heales, JH cross, and S Sisodiya. An examination of biochemical parameters and their association with response to ketogenic dietary therapies. Epilepsia. 2017:58:893-900.

22. Dahlin M, Elfving Å, Ungerstedt U, Åmark P. The ketogenic diet influences the levels of excitatory and inhibitory amino acids in the CSF in children with refractory epilepsy. Epilepsy Res. 2005;64:115-25.

23. Dahlin M, Månsson J-E, Åmark P. CSF levels of dopamine and serotonin, but not norepinephrine, metabolites are influenced by the ketogenic diet in children with epilepsy. Epilepsy Res. 2012;99:132-8.

24. Sariego-Jamardo A, García-Cazorla A, Artuch R, Castejón E, García-Arenas $D$, Molero-Luis M, et al. Efficacy of the ketogenic diet for the treatment of refractory childhood epilepsy: cerebrospinal fluid neurotransmitters and amino acid levels. Pediatr Neurol. 2015;53:422-6.

25. Xu X-p, Erichsen D, Börjesson SI, Dahlin M, Åmark P, Elinder F. Polyunsaturated fatty acids and cerebrospinal fluid from children on the ketogenic diet open a voltage-gated K channel: A putative mechanism of antiseizure action. Epilepsy Res. 2008;80:57-66.

26. Wei C, Li Y, Yao H, Liu H, Zhang X, Guo R. A metabonomics study of epilepsy in patients using gas chromatography coupled with mass spectrometry. Mol BioSyst. 2012;8:2197-204.

27. Wang H, Liang S, Wang M, Gao J, Sun C, Wang J, et al. Potential serum biomarkers from a metabolomics study of autism. J Psychiatry Neurosci. 2016:41:27-37.

28. O'Sullivan A, Gibney MJ, Brennan L. Dietary intake patterns are reflected in metabolomic profiles: potential role in dietary assessment studies. Am J Clin Nutr. 2011;93:314-21.

29. Douris N, Melman T, Pecherer JM, Pissios P, Flier JS, Cantley LC, et al. Adaptive changes in amino acid metabolism permit normal longevity in mice consuming a low-carbohydrate ketogenic diet. Biochim Biophys Acta. 2015;1852:2056-65.

30. Klein MS, Newell C, Bomhof MR, Reimer RA, Hittel DS, Rho JM, et al. Metabolomic modeling to monitor host responsiveness to gut microbiota manipulation in the $B_{T B R}{ }^{T+t / j} / 3$ mouse. J Proteome Res. 2016;15:1143-50

31. Berg AT, Berkovic SF, Brodie MJ, Buchhalter J, Cross JH, van Emde Boas W, et al. Revised terminology and concepts for organization of seizures and epilepsies: report of the ILAE Commission on Classification and Terminology, 2005-2009. Epilepsia. 2010;51:676-85.

32. Trabi M, Keller MD, Jonsson NN. NMR-based metabonomics of bovine blood: an investigation into the effects of long term storage on plasma samples. Metabolomics. 2013;9:1041-7.

33. Suhre K, Shin SY, Petersen AK, Mohney RP, Meredith D, Wagele B, et al. Human metabolic individuality in biomedical and pharmaceutical research. Nature. 2011;477:54-60.
34. Swink TD, Vining EP, Freeman JM. The ketogenic diet: 1997. Adv Pediatr Infect Dis. 1997:44:297-39.

35. Casazza JP, Felver ME, Veech RL. The metabolism of acetone in rat. J Biol Chem. 1984;259:231-6.

36. Juraschek JP, Miller ER III, Appel L, Christenson RH, Sacks FM, Selvin E. Effects of dietary carbohydrates on 1,5-anhydroglucitol in a population without diabetes: Results from the OmniCarb Trial. Diabet Med. 2017; $34: 1407$

37. DeVivo, DC, MP Leckie, JS Ferrendelli, DB McDougal, and Jr. Chronic ketosis and cerebral metabolism. Ann Neurol 1978;3:331-337.

38. Courchesne-Loyer A, Croteau E, Castellano C-A, St-Pierre V, Hennebelle $\mathrm{M}$, Cunnane SC. Inverse relationship between brain glucose and ketone metabolism in adults during short-term moderate dietary ketosis: a dual tracer quantitative positron emission tomography study. J Cereb Blood Flow Metab. 2017;37:2485-93.

39. Ruderman NB, Ross PS, Berger M, Goodman MN. Regulation of glucose and ketone-body metabolism in brain of anaesthetized rats. Biochem J. 1974;138:1-10.

40. Hasselbalch SG, Madsen PL, Hageman LP, Olsen KS, Justesen N, Holm S, Paulson OB. Changes in cerebral blood flow and carbohydrate metabolism during acute hyperketonemia. Am J Physiol Endocrinol Metab. 1996;270:E746-E51.

41. Wang X, Lui Q, Zhou J, Wu X, Zhu Q. $\beta$ hydroxybutyrate levels in serum and cerebrospinal fluid under ketone body metabolism in rats. Exp Anim. 2017:66:177-82.

42. White H, Venkatesh B, Jones M, Worrall S, Chuah T, Ordonez J. Effect of a hypertonic balanced ketone solution on plasma, CSF and brain beta-hydroxybutyrate levels and acid-base status. Intensive Care Med. 2013:39:727-33.

43. Giménez-Cassina A, Martínez-François JR, Fisher JK, Szlyk B, Polak K, Wiwczar J, et al. BAD-dependent regulation of fuel metabolism and $\mathrm{K}_{\text {ATP }}$ channel activity confers resistance to epileptic seizures. Neuron. 2012;74:719-30.

44. Juge N, Gray JA, Omote H, Miyaji T, Inoue T, Hara C, et al. Metabolic control of vesicular glutamate transport and release. Neuron. 2010;68:99-112.

45. Garbow JR, Doherty JM, Schugar RC, Travers S, Weber ML, Wentz AE, et al. Hepatic steatosis, inflammation, and ER stress in mice maintained long term on a very low-carbohydrate ketogenic diet. Am J Physiol Gastrointest Liver Physiol. 2011;300:G956-G67.

46. Elamin M, Ruskin DN, Masino SA, Sacchetti P. Ketogenic diet modulates $\mathrm{NAD}^{+}$-dependent enzymes and reduces DNA damage in hippocampus. Front Cell Neurosci. 2018:12:263.

47. Shimazu T, Hirschey MD, Newman J, He W, Shirakawa K, Le Moan N, et al. Suppression of oxidative stress by b-hydroxybutyrate, an endogenous histone deacetylase inhibitor. Science. 2013;339:211-4.

48. McQuarrie I, Keith HM. Epilepsy in children: relationship of variations in the degree of ketonuria to occurrence of convulsions in epileptic children on ketogenic diets. Am J Dis Child. 1927;34:1013-29.

49. Wilder RM, Winter MD. The threshold of ketogenesis. J Biol Chem. 1922;52:393-401

50. Gilbert DL, Pyzik PL, Freeman JM. The ketogenic diet: seizure control correlates better with serum beta-hydroxybutyrate than with urine ketones. J Child Neurol. 2000:15:787-90.

51. Huttenlocher PR. Ketonemia and seizures: metabolic and anticonvulsant effects of two ketogenic diets in childhood epilepsy. Pediatr Res. 1976;10:536-40.

52. Lambrechts DAJE, de Kinderen RJA, Vles JSH, de Louw AJA, Aldenkamp AP, Majoie HJM. A randomized controlled trial of the ketogenic diet in refractory childhood epilepsy. Acta Neuro Scand. 2016;135:678.

53. Fraser DA, Thoen J, Bondhus S, Haugen M, Reseland JE, Djøseland O, et al. Reduction in serum leptin and IGF-1 but preserved T-lymphocyte numbers and activation after a ketogenic diet in rheumatoid arthritis patients. Clin Exp Rheumatol. 2000;18:209-14.

54. Kang H-C, Lee HS, You SJ, Kang DC, Ko T-S, Kim HD. Use of a modified Atkins diet in intractable childhood epilepsy. Epilepsia. 2007;48:182-6.

55. Kossoff EH, McGrogan JR, Bluml RM, Pillas DJ, Rubenstein JE, Vining EP. A modified Atkins diet is effective for the treatment of intractable pediatric epilepsy. Epilepsia. 2006;47:421-4.

56. van Delft R, Lambrechts D, Verschuure P, Hulsman J, Majoie M. Blood beta-hydroxybutyrate correlates better with seizure reduction due to ketogenic diet than do ketones in the urine. Seizure. 2010;19:36-9. 
57. Neal EG, Chaffe H, Schwartz RH, Lawson MS, Edwards N, Fitzsimmons $G$, et al. A randomized trial of classical and medium-chain triglyceride ketogenic diets in the treatment of childhood epilepsy. Epilepsia. 2009;50:1109-17.

58. Kawamura M Jr, Ruskin DN, Masino SA. Metabolic autocrine regulation of neurons involves cooperation among pannexin hemichannels, adenosine receptors and $K_{\text {ATP }}$ channels. J Neurosci. 2010;30:3886-95.

59. Kawamura M Jr, Ruskin DN, Geiger JD, Boison D, Masino SA. Ketogenic diet sensitizes glucose control of hippocampal excitability. J Lipid Res. 2014;55:2254-60.

60. Masino SA, Li T, Theofilas P, Sandau U, Ruskin DN, Fredholm BB, et al. A ketogenic diet suppresses seizures in mice through adenosine $A_{1}$ receptors. J Clin Invest. 2011;121:2679-83.

61. Stafstrom CE, Ockuly JC, Murphree L, Valley MT, Roopra A, Sutula TP. Anticonvulsant and antiepileptic actions of 2-deoxy-D-glucose in epilepsy models. Ann Neurol. 2009;65:435-47.

62. Gasior M, Yankura J, Hartman AL, French A, Rogawski MA. Anticonvulsant and proconvulsant actions of 2-deoxy-D-glucose. Epilepsia. 2010;51:1385-94.

63. Dallérac G, Moulard J, Benoist J-F, Rouach S, Auvin S, Guilbot A, et al. Non-ketogenic combination of nutritional strategies provides robust protection against seizures. Sci Rep. 2017;7:5496.

64. Karimzadeh P, Sedighi M, Beheshti M, Azargashb E, Ghofrani M, Abdollahe-Gorgi F. Low glycemic index treatment in pediatric refractory epilepsy: the first Middle East report. Seizure. 2014;23:570-2.
65. Meidenbauer JJ, Roberts MF. Reduced glucose utilization underlies seizure protection with dietary therapy in epileptic EL mice. Epilepsy Behav. 2014;39:48-54

66. Hadera, MG, T McDonald, OB Smeland, TW Meisingset, H Eloqayli, S Jaradat, et al. Modification of astrocyte metabolism as an approach to the treatment of epilepsy: Triheptanoin and acetyl-L-carnitine. Neurochem Res. 2015;in press.

67. Traina $G$. The neurobiology of acetyl-L-carnitine. Front Biosci. 2016;21:1314-29.

68. Schaub A, Fütterer A, Pfeffer K. PUMA-G, an IFN-gamma-inducible gene in macrophages is a novel member of the seven transmembrane spanning receptor superfamily. Eur J Immunol. 2001;31:3714-25.

69. Maitre M, Hechler V, Vayer P, Gobaille S, Cash CD, Schmitt M, Bourguignon JJ. A specific gamma-hydroxybutyrate receptor ligand possesses both antagonistic and anticonvulsant properties. J Pharmacol Exp Ther. 1990;255:657-63.

70. Brown AJ. Low-carb diets, fasting and euphoria: is there a link between ketosis and g-hydroxybutyrate (GHB)? Med Hypotheses. 2007;68:268-71.

\section{Publisher's Note}

Springer Nature remains neutral with regard to jurisdictional claims in published maps and institutional affiliations.
Ready to submit your research? Choose BMC and benefit from:

- fast, convenient online submission

- thorough peer review by experienced researchers in your field

- rapid publication on acceptance

- support for research data, including large and complex data types

- gold Open Access which fosters wider collaboration and increased citations

- maximum visibility for your research: over $100 \mathrm{M}$ website views per year

At BMC, research is always in progress.

Learn more biomedcentral.com/submissions 